\title{
TRATAMENTO CONSERVADOR DO CARCINOMA DO CANAL ANAL
}

\author{
CONSERVATIVE TREATMENT OF ANAL CANAL CARCINOMA
}

\author{
Ricardo Akiyoshi Nakamura ${ }^{1}$; Robson Ferrigno ${ }^{2}$; João Victor Salvajoli²; \\ Inês Nobuko Nishimoto ${ }^{3}$; Waldec Jorge David Filho ${ }^{4}$; Ademar Lopes, TCBC-SP5
}

\begin{abstract}
RESUMO: Objetivos: Relatar os resultados do tratamento conservador do carcinoma de canal anal com radioterapia e quimioterapia do Centro de Tratamento e Pesquisa Hospital do Câncer A.C. Camargo. Método: De março de 1993 a dezembro de 2001, 47 pacientes com diagnóstico histológico de carcinoma do canal anal foram tratados de forma conservadora. A dose mediana de radioterapia na pelve e no tumor primário foi respectivamente de 45 e $55 \mathrm{~Gy}$. A quimioterapia foi realizada com 5 Fluorouracil e Mitomicina-C, com doses medianas de $1000 \mathrm{mg} / \mathrm{m}^{2}$ por quatro dias e $10 \mathrm{mg} / \mathrm{m}^{2}$ por ciclo, respectivamente. Trinta e oito $(80,8 \%)$ pacientes não receberam radioterapia em região inguinal. $\mathrm{O}$ tempo de seguimento mediano foi de 40 meses (oito dias a 116 meses). Resultados: A resposta completa foi alcançada em 40 pacientes $(85,1 \%)$. O controle local foi obtido em 31 (66\%), e a função esfincteriana foi preservada em $38(80,9 \%)$ casos. Metástases à distância foram detectadas em sete (14,9\%) pacientes. A sobrevida global e sobrevida livre de doença em cinco anos foram de $61,5 \%$ e $50,1 \%$, respectivamente. A sobrevida global e a sobrevida livre de doença em cinco anos para os pacientes que tiveram controle local foram 77,8\% $(p<0,001)$ e $74,4 \%$ $(p<0,001)$. A sobrevida global e livre de doença em cinco anos para os pacientes com linfonodo inguinal clinicamente tumoral foi de $70,7 \%$ e $56,7 \%$, respectivamente $(p=0,0085$ e $p=0,0207)$. Doze $(25,5 \%)$ pacientes necessitaram de interrupção temporária do tratamento. Cinco pacientes tiveram complicações crônicas leves. Conclusão: O tratamento realizado foi efetivo tanto para preservação do esfíncter anal quanto para controle local de doença. A presença de linfonodo inguinal clinicamente tumoral e a ausência de recidiva foram os principais fatores prognósticos para sobrevida global e sobrevida livre de doença. A taxa relativamente alta de recidiva em região inguinal sugere a necessidade de radioterapia eletiva nessa região (Rev. Col. Bras. Cir. 2005; 32(1): 23-31).
\end{abstract}

Descritores: Carcinoma; Ânus; Neoplasias do ânus; Radioterapia; Quimioterapia.

\section{INTRODUÇÃO}

O câncer do canal anal é neoplasia pouco freqüente e compreende $1 \%$ a $2 \%$ dos tumores do intestino grosso ${ }^{1}$. Sua incidência tem aumentado substancialmente após a década de 80, provavelmente em função da maior incidência de transmissão sexual do papiloma vírus humano². Cerca de $75 \%$ a $80 \%$ destes tumores são carcinomas espinocelulares $(\mathrm{CEC})^{3}$, e há uma predominância no sexo feminino de 2:1 a 5:1 na dependência da série considerada ${ }^{4,5}$. A doença acomete principalmente pacientes na sexta e sétima décadas de vida $^{6}$.

A distinção anatômica entre o canal anal e a margem anal é necessária, pois a história natural do câncer nestas regiões é diferente. Tumores de margem anal comportam-se como de pele e devem ser classificados e tratados como tal ${ }^{7}$. No passado, vários autores definiram o limite distal do canal anal como a linha denteada e todos os tumores abaixo eram classificados como câncer da margem anal ${ }^{8-11}$. Outros autores definiram o limite distal do canal anal como a borda anal ${ }^{12-14}$. Para esclarecer o assunto, a "American Joint Committee on Cancer" (AJCC) e a "Union Internationale Contra le Cancer" (UICC) estabeleceram que o canal anal estende-se do anel anorretal ( $2 \mathrm{~cm}$ acima da linha denteada) à borda anal ${ }^{15,16} \mathrm{e}$, no atual sistema de estadiamento, o consenso foi mantido ${ }^{17,18}$.

A abordagem terapêutica do carcinoma de canal anal evoluiu muito nos últimos 30 anos em função dos resultados de preservação da função esfincteriana. Até a década de 1970, o tratamento padrão era a ressecção abdominal perineal com colostomia definitiva. Em 1974, Nigro et al. ${ }^{19}$ publicaram os resultados preliminares de três pacientes tratados com quimioterapia com 5-fluorouracil (5-FU) e mitomicina $\mathrm{C}(\mathrm{MMC})$ concomitantes à radioterapia de forma pré-operatória. Foi evidenciada resposta completa após o procedimento cirúrgico em dois pacientes e ausência de recorrência de doença 15 meses após o tratamento no paciente que recusou cirurgia. Desde então, numerosos estudos clínicos têm sido realizados com rádio e quimioterapia em diversos esquemas para o carcinoma de canal anal com resultados terapêuticos considerados satisfatórios. A cirurgia tem sido reservada para o resgate em eventual falha terapêutica ou para os casos com doença destrutiva do canal anal $^{6,20}$.

A presente série tem o objetivo de relatar os resultados do tratamento conservador do carcinoma do canal anal com rádio e quimioterapia realizados no Centro de Tratamento e Pesquisa Hospital do Câncer A. C. Camargo.

1. Residente do Departamento de Radioterapia

2. Departamento de Radioterapia

3. Estatística do Centro de Estudos

4. Departamento de Oncologia Clínica

5. Departamento de Cirurgia Pélvica

Recebido em 24/06/2004

Aceito para publicação em 02/09/2004

Trabalho realizado no Centro de Tratamento e Pesquisa Hospital do Câncer A.C. Camargo - São Paulo - SP. 


\section{MÉTODO}

De março de 1993 a dezembro de 2001, 47 pacientes com diagnóstico histológico de carcinoma epidermóide ou basalóide de canal anal foram tratados no Centro de Tratamento e Pesquisa Hospital do Câncer A. C. Camargo (CTPHCACC) com radioterapia (RT) associada ou não à quimioterapia (QT), dependendo do estádio clínico (EC). Os pacientes foram avaliados através da história clínica e exame físico completos, seguidos de retossigmoidoscopia, tomografia computadorizada de abdome e pelve, radiografia do tórax e biópsia da lesão para análise histológica em todos os pacientes. Após o diagnóstico histopatológico, os pacientes foram estadiados conforme os critérios estabelecidos pela AJCC/ UICC $^{17,18}$, que os dividem segundo as categorias T (extensão do tumor primário), $\mathrm{N}$ (ausência ou presença e extensão de metástases linfonodais regionais) e M (ausência ou presença de metástases à distância), e os agrupam em seis classes de estádios clínicos.

A radioterapia foi realizada $\mathrm{em}$ aceleradores lineares com energias de $4 \mathrm{MeV}$ ou $6 \mathrm{MeV}$. Os campos de radiação tiveram como limites cranial a transição L5-S1, e caudal $2 \mathrm{~cm}$ abaixo da extensão distal do tumor primário e laterais, $2 \mathrm{~cm}$ lateralmente à margem óssea da parede pélvica verdadeira ${ }^{21,22}$. A complementação de dose foi elaborada de acordo com o volume do tumor primário. Quando a radioterapia inguinal foi realizada, a técnica utilizada foi a descrita por Kalend et $a l^{23}$. Através desta técnica, coloca-se um bloco de transmissão no campo anterior com o mesmo formato do campo posterior. $\mathrm{O}$ bloco possui finalidade de liberar no meio da espessura do paciente, a metade da dose ( $90 \mathrm{cGy}$ ) que é prescrita a 2 ou $3 \mathrm{~cm}$ de profundidade (180 cGy) nas regiões inguinais. A metade remanescente da dose (90 cGy) é liberada pelo campo posterior no meio do diâmetro ântero-posterior do paciente. A radioterapia em região inguinal foi considerada eletiva quando não havia linfonodos inguinais neoplásicos, e terapêutica quando os linfonodos inguinais estavam comprometidos.

A QT foi realizada com 5-Fluorouracil (5-FU) em infusão contínua intravenosa nos quatro primeiros e quatro últimos dias da RT (D1 a D4 e D27 a D30), e Mitomicina-C (MMC) em infusão intravenosa no primeiro dia da RT e, se houvesse condições clínicas e a dose total não excedesse $20 \mathrm{mg} / \mathrm{m}^{2}$, no D29 da RT.

Trinta dias após o tratamento, todos os pacientes foram submetidos a exame proctológico com biópsia para avaliação de resposta. A resposta foi considerada completa se a biópsia fosse negativa e não houvesse lesão residual. Se a biópsia fosse positiva e não houvesse crescimento da lesão, a biópsia era repetida em dois meses. Se a biópsia ainda fosse positiva, a resposta foi considerada parcial e o paciente era submetido à cirurgia radical de resgate. Se a biópsia inicial era positiva e houvesse crescimento da lesão, foi considerado progressão de doença e o paciente era submetido à amputação abdominoperineal (se houvesse condições clínicas) ${ }^{24}$.

Nas respostas completas, as consultas de retorno foram trimestrais nos dois primeiros anos, semestrais do terceiro ao quinto ano, e anuais após. Nos retornos, as queixas eram avaliadas e o exame físico era realizado de forma completa, incluindo toque retal e palpação da região inguinal. Radiografia do tórax e tomografia computadorizada da pelve foram solicitados a cada seis meses nos cinco primeiros anos e, anualmente, a retossigmoidoscopia era realizada após verificação da resposta completa ${ }^{25}$.

Os efeitos tóxicos da radioterapia foram avaliados através de escalas de morbidades agudas e tardias elaboradas pelo "Radiation Therapy Oncology Group" (RTOG) e pela "European Organization for Reserch and Treatment of Cancer" $(\text { EORTC })^{26}$, conforme Tabelas 1 e 2, respectivamente. Os efeitos tóxicos da quimioterapia foram avaliados pela escala da "Eastern Cooperative Oncology Group" (ECOG) ${ }^{27}$ conforme Tabela 3.

Para a análise estatística, foram realizadas estatísticas descritivas através de medidas de tendência central, de variabilidade e distribuição de frequiências. Os cálculos das probabilidades de sobrevida global e sobrevida livre de doença dos pacientes foram realizados pelo método atuarial de Kaplan e $\mathrm{Meir}^{28}$. As comparações entre a distribuição de sobrevida nas categorias de uma mesma variável foram feitas através do teste logrank, também conhecido como COXMANTEL. Para a comparação de variáveis categóricas, foram realizados testes do qui-quadrado $\left(\mathrm{c}^{2}\right)$, ou teste Exato de Fisher, dependendo da casuística, ou quando as freqüências esperadas foram menores que cinco. Foram considerados significativos os valores de $p<0,05$.

\section{RESULTADOS}

O tempo de seguimento variou de oito dias a 124 meses, com mediana de 40 meses e média (dp) de 46 (31) meses. A idade dos pacientes variou de 36 a 77 anos, com mediana de 56 anos. Houve uma predominância do sexo feminino de aproximadamente $4: 1$, sendo $36(76,6 \%)$ mulheres e 11 $(23,4 \%)$ homens. A distribuição dos pacientes por estádio clínico foi: I - três (6,4\%), II - 27 (57,5\%), IIIA - oito (17\%) e IIIB - nove $(19,1 \%)$.

Quarenta e dois $(89,4 \%)$ pacientes foram tratados com RT e QT concomitantes. Dois pacientes não tiveram condições clínicas para QT e três eram EC I não tendo indicação de QT. A dose de RT na pelve variou de 9 Gy a 50,40 Gy, com mediana de 45 Gy e a dose no tumor primário variou de 9 Gy a $68,40 \mathrm{~Gy}$, com mediana de $55 \mathrm{~Gy}$. Trinta e oito $(80,8 \%)$ pacientes não receberam RT em região inguinal. A dose mediana de 5-FU em cada ciclo foi de $1000 \mathrm{mg} / \mathrm{m}^{2} /$ dia por quatro dias, e a dose total mediana de $\mathrm{MMC}$ foi de $10 \mathrm{mg} / \mathrm{m}^{2}$ por ciclo.

A resposta clínica, avaliada após 30 dias do término do tratamento, foi considerada completa em 40 pacientes $(85,1 \%)$. A recidiva loco-regional ocorreu em 16 (34\%) pacientes, e quatro foram resgatados cirurgicamente e encontram-se controlados. A função esfincteriana foi preservada em 38 pacientes $(80,9 \%)$. O controle local para os estádios clínicos I e II, e IIIA e IIIB foram respectivamente de $67,7 \%$ e $32,3 \%$ ( $p=$ $0,528)$, e para os pacientes $\mathrm{T} 1 \mathrm{e} \mathrm{T} 2$, e T3 e T4 foram respectivamente de $74,2 \%$ e $25,8 \%$ ( $p=0,506)$. A sobrevida livre de doença em cinco anos foi significativamente maior para os pacientes T1 e T2 $(60,3 \%, p=0,0111)$. 
De acordo com a dose de RT na pelve, a taxa de controle local nos pacientes que receberam até 44 Gy e acima desta dose foi de $12,9 \%$ (quatro pacientes) e $87,1 \%$ (27 pacientes), respectivamente $(p=0,024)$. Metástases à distância foram detectadas em sete $(14,9 \%)$ pacientes, sendo um $(2,1 \%)$ em retroperitônio, quatro $(8,5 \%)$ em fígado e dois $(4,2 \%)$ em pulmão.

Trinta e oito $(80,8 \%)$ pacientes não receberam RT em região inguinal. Desses, cinco (15\%) evoluíram com recidiva na região inguinal, sendo que um era inicialmente estádio clínico II e quatro estádio clínico IIIB. Nenhum dos quatro pacientes que receberam RT eletiva em região inguinal teve recidiva nesta região. Dos cinco $(11,6 \%)$ pacientes que foram submetidos a RT inguinal terapêutica, um não teve a doença controlada nesta região.

No final deste estudo, vinte e dois $(46,8 \%)$ pacientes encontram-se vivos sem evidência de doença, um $(2,1 \%)$ está vivo com evidência de doença, 12 (25,5\%) faleceram pela doença, dois $(4,3 \%)$ faleceram por segundo tumor primário, quatro $(8,5 \%)$ faleceram pelo tratamento, dois $(4,3 \%)$ estão perdidos de seguimento, e quatro $(8,5 \%)$ foram resgatados localmente por cirurgia e encontram-se controlados. As probabilidades de sobrevida global e de sobrevida livre de doença em cinco anos foram de $61,5 \%$ e $50,1 \%$, respectivamente. Agrupando os EC I e II,e IIIA e IIIB, a probabilidade de sobrevida global em cinco anos foi $69,5 \%$ e 49,5 respectivamente $(p=$ 0,0763). A sobrevida livre de doença em cinco anos para este mesmo agrupamento foi $59 \%$ e 33,4\% ( $p=0,0543)$. Em relação à sobrevida global em 5 anos, os fatores prognósticos que alcançaram significância estatística foram (Tabela 4) presença ou ausência de linfonodo inguinal tumoral $(p=0,0085)$, RT em pelve maior ou menor que $45 \mathrm{~Gy}(p=0,0110)$, resposta inicial ao tratamento completa ou não $(p<0,001)$ e controle local ou não com o tratamento conservador $(p<0,001)$. Para sobrevida livre de doença em cinco anos, os fatores prognósticos estatisticamente significativos foram (Tabela 4) tamanho do tumor (T1 e T2 ou T3 e T4, $p=0,0111$ ), presença ou não de linfonodo inguinal tumoral $(p=0,0207)$, resposta inicial ao tratamento completa ou não $(p<0,001)$, esfíncter preservado ou não $(p<$ $0,001)$ e controle local ou não ( $p<0,001)$. Em relação ao controle local, os fatores que alcançaram significância estatística foram (Tabela 5) RT em pelve menor que 45Gy ou não ( $p=$ 0,024). A função esfincteriana foi preservada em $38(80,9 \%)$ pacientes.

O tempo de tratamento variou de oito a 129 dias, com mediana de 49 dias. A sobrevida global em cinco anos para os pacientes que foram tratados em até 49 dias foi de $63,7 \%$, e para os que foram tratados em mais de 49 dias foi de $59,8 \%$ $(p=0,5533)$. O controle local em cinco anos para os pacientes que foram tratados em até 49 dias foi de $51,2 \%$, enquanto que para os pacientes tratados em mais de 49 dias foi de $49,4 \%$ $(p=0,4351)$.

As variáveis clínicas, idade, sexo e presença ou ausência de linfonodos clinicamente tumorais não foram estatisticamente significativas para o controle local, sobrevida global e livre de doença em cinco anos.

Com relação ao tratamento, a realização ou não de RT inguinal, a dose de RT maior ou menor que 55 Gy no tumor primário e a necessidade ou não de suspensão do tratamento não influenciaram a sobrevida global, a sobrevida livre de doença ou o controle local.

Quanto à toxicidade aguda, todos os 47 pacientes apresentaram radiodermite durante o tratamento, sendo um $(2,1 \%)$ grau $1,10(21,3 \%)$ grau 2 e $35(74,5 \%)$ grau 3 segundo graduação de toxicidade aguda da RTOG/EORTC (Tabela 1). Leucopenia foi observada em $13(27,6 \%)$ dos 47 pacientes, dois $(5 \%)$ grau 2 , dois (5\%) grau 3 e cinco (12\%) grau 4 pela graduação de toxicidade da ECOG (Tabela 3). Quatro pacientes faleceram durante o tratamento por leucopenia febril (10\%). Um $(2 \%)$ paciente apresentou síndrome de mão pé por $5-\mathrm{FU}$. Vinte e três $(48,9 \%)$ dos 47 pacientes tiveram diarréia aguda, $13(27,6 \%)$ vômitos, e sete $(14,9 \%)$ disúria. A interrupção temporária do tratamento por toxicidade aguda foi necessária em $12(28 \%)$ pacientes, com período de interrupção variando de 10 a 34 dias, com mediana de 15 dias. Dois (5\%) pacientes tiveram o tratamento suspenso por outras causas. Quanto às complicações crônicas, quatro pacientes tiveram toxicidade retal grau 1 e um paciente toxicidade cutânea grau 2 pela classificação RTOG/EORTC (Tabela 2).

\section{DISCUSSÃO}

Até o fim da década de 1970, o tratamento convencional para o carcinoma de canal anal foi a ressecção abdominoperineal ${ }^{7}$, a qual exigia o sacrifício do esfíncter anal e resultava em $27 \%$ a $47 \%$ de recidiva local $^{1,29}$ e taxas de sobrevida global em cinco anos de $37 \%$ a $71 \%{ }^{30}$. Estudos de Nigro 19, 31 iniciados na década de 70, no entanto, questionavam a necessidade do sacrifício do esfíncter anal para esses pacientes, e culminaram, em 1984, em um programa definitivo de tratamento conservador do carcinoma de canal anal através da análise retrospectiva de 104 pacien$\operatorname{tes}^{32}$.

Embora não haja ensaios clínicos controlados e aleatorizados que comparem cirurgia com radioterapia exclusiva ou cirurgia com tratamento combinado com rádio e quimioterapia, provavelmente devido à raridade dos carcinomas de canal anal e da forte evidência clínica vinda de diversos estudos fase II de tratamento conservador dos últimos 20 anos, a modalidade de tratamento conservador do carcinoma de canal anal com rádio e quimioterapia tornou-se o padrão para esses pacientes, sendo a cirurgia reservada para o resgate, em caso de falha ou para os casos de doença grave e destrutiva do esfíncter anal $\mathrm{l}^{6,7,20,33}$.

Dois estudos controlados aleatorizados europeus, um do EORTC ${ }^{34}$ e outro do $\mathrm{UKCCCR}^{35}$, compararam rádio e quimioterapia concomitantes com radioterapia exclusiva e comprovaram a superioridade do tratamento combinado. Embora nenhum deles tenha demonstrado vantagem na sobrevida global com o tratamento combinado, os melhores índices de controle local e sobrevida livre de colostomia foram definitivos no estabelecimento da modalidade combinada como o tratamento padrão para o carcinoma de canal anal ${ }^{7}$.

A MMC é um antibiótico extraído da espécie Streptomyces ${ }^{36}$ e utilizado no tratamento do câncer de mama $^{37}$, de nasofaringe ${ }^{38}$ e nos tumores gastrointestinais ${ }^{39}$. 
Tabela 1 - Escala do RTOG/EORTC para graduação da morbidade aguda da radioterapia.

\begin{tabular}{lllll}
\hline Órgão/Tecido & Grau 1 & Grau 2 & Grau 3 & Grau 4 \\
\hline Pele & $\begin{array}{l}\text { Eritema folicular fraco } \\
\text { ou apagado, epilação, } \\
\text { descamação seca, } \\
\text { diminuição da sudorese }\end{array}$ & $\begin{array}{l}\text { Eritema brando ou claro, } \\
\text { descamação úmida em } \\
\text { placas, edema moderado }\end{array}$ & $\begin{array}{l}\text { Descamação úmida } \\
\text { confluente, além das } \\
\text { dobras da pele, } \\
\text { edema em “casca } \\
\text { de laranja” }\end{array}$ & $\begin{array}{l}\text { Ulceração, hemorragia, } \\
\text { necrose }\end{array}$ \\
$\begin{array}{l}\text { Gastrintestinal } \\
\text { inferior }\end{array}$ & $\begin{array}{l}\text { Aumento da freqüência } \\
\text { dos hábitos intestinais } \\
\text { não necessitando de } \\
\text { medicação e desconforto } \\
\text { retal não necessitando } \\
\text { de analgésicos }\end{array}$ & $\begin{array}{l}\text { Diarréia necessitando } \\
\text { de medicação e dor } \\
\text { retal ou abdominal } \\
\text { necessitando de } \\
\text { analgésicos }\end{array}$ & $\begin{array}{l}\text { Diarréia necessitando } \\
\text { de suporte parenteral, } \\
\text { mucosite grave, perda } \\
\text { de sangue ou } \\
\text { distensão abdominal }\end{array}$ & $\begin{array}{l}\text { Obstrução aguda ou } \\
\text { subaguda, fístula ou } \\
\text { perfuração, sangramento } \\
\text { requerendo transfusão } \\
\text { ou dor requerendo } \\
\text { sonda nasogástrica } \\
\text { ou cirurgia }\end{array}$ \\
\hline
\end{tabular}

Em função de sua alta toxicidade, principalmente hematológica, e, apesar do estudo retrospectivo do "Princess Margaret Hospital"40 sugerir a importância da MMC, o seu papel foi estabelecido somente após os resultados reportados pelo "Intergroup" 41 em 1996, que demonstrou que o grupo de pacientes que recebeu MMC obteve taxas maiores de sobrevida livre de colostomia (71\% versus $59 \% ; p=0,014)$, sobrevida livre de doença ( $73 \%$ versus $51 \%$; $p=0,0003$ ), e menor taxa de colostomia ( $9 \%$ versus $22 \%$, $p=0,002)$. Embora a sobrevida global não tenha aumentado significativamente, o aumento na sobrevida livre de colostomia faz da MMC componente necessário do regime combinado. Com isso, a abordagem terapêutica mais comum nos Estados Unidos para o carcinoma de canal anal é o tratamento combinado com radioterapia, 5-FU e MMC nos moldes deste estudo ${ }^{7}$.

No entanto, esforços têm sido realizados no intuito de remover a MMC do esquema combinado em função de sua alta toxicidade. Vários estudos não aleatorizados baseados em cisplatina (CDDP) com resultados animadores têm sido reportados ${ }^{7,42-50}$. Embora utilizem doses maiores de radioterapia, com índices de resposta completa similares $(85 \%$, variando de $80 \%$ a $96 \%$ ), os dados sugerem que as taxas médias de controle local ( $87 \%$, variando de $80 \%$ a $94 \%$ ), sobrevida livre de colostomia ( $71 \%$, variando de $56 \%$ a $86 \%)$, e sobrevida global ( $89 \%$, variando de $84 \%$ a $94 \%)$ podem ser maiores do que os regimes baseados em MMC, sem a alta toxicidade desta. Entretanto, deve-se enfatizar que as diferenças encontradas podem ser consequientes a outros fatores como doses maiores de radiação e não aleatorização dos pacientes.

O "Intergroup" tem desenvolvido um ensaio clínico (RTOG 98-11) em que 650 pacientes com carcinoma de canal anal estão sendo aleatorizados para receber o esquema tradicional com radioterapia, 5-FU $\left(1.000 \mathrm{mg} / \mathrm{m}^{2} / \mathrm{dia}\right.$ em infusão contínua de D1 a D4, e de D29 a D32) e MMC (10mg/m² nos dias 1 e 29) concomitantes, ou dois ciclos de quimioterapia de indução com CDDP $75 \mathrm{mg} / \mathrm{m}^{2}$ intravenosa em 60 minutos nos dias 1 e 29, e 5-FU $1000 \mathrm{mg} / \mathrm{m}^{2} /$ dia em infusão contínua dos dias 1 a 4 e 29 a 32, seguidos por radioterapia, 5-FU e CDDP concomitantes.

A intensificação de dose de radioterapia tem sido realizada em diversas séries procurando aumentar o controle local. As séries do "Massachusetts General Hospital" ${ }^{51}$ e do "M. D. Anderson Hospital" 52 sugeriram um aumento do controle local com a intensificação de dose (59,4 Gy de dose

Tabela 2 - Escala do RTOG/EORTC para morbidade tardia da radioterapia. Óbito por complicação é considerado grau 5.

\begin{tabular}{lllll}
\hline Órgão/Tecido & Grau 1 & Grau 2 & Grau 3 & Grau 4 \\
\hline Intestino & $\begin{array}{l}\text { Diarréia branda, leves } \\
\text { cólicas, movimentação } \\
\text { intestinal 5 vezes ao dia, } \\
\text { leve descarga retal ou } \\
\text { sangramento }\end{array}$ & $\begin{array}{l}\text { Diarréia moderada e cólica, } \\
\text { movimentação intestinal } \\
5 \text { vezes ao dia, excessivo } \\
\text { muco retal ou } \\
\text { sangramento repetitivo }\end{array}$ & $\begin{array}{l}\text { Obstrução ou sangramento } \\
\text { requerendo cirurgia }\end{array}$ & $\begin{array}{l}\text { Necrose, perfuração } \\
\text { ou fístulas }\end{array}$ \\
Bexiga & $\begin{array}{l}\text { Atrofia epitelial leve, } \\
\text { telangiectasia branda ou } \\
\text { hematúria microscópica }\end{array}$ & $\begin{array}{l}\text { Freqüência moderada, } \\
\text { telangiectasia generalizada } \\
\text { e hematúria microscópica } \\
\text { retitiva }\end{array}$ & $\begin{array}{l}\text { Frequiência elevada } \\
\text { e disúria, telangiectasia } \\
\text { generalizada grave, } \\
\text { hematúria freqüente ou } \\
\text { redução na capacidade } \\
\text { vesical (<150cc) }\end{array}$ & $\begin{array}{l}\text { Necrose, bexiga } \\
\text { contraída (capacidade } \\
<100 c c) \text { ou cistite } \\
\text { hemorrágica grave }\end{array}$ \\
\end{tabular}


Tabela 3 - ECOG - Critério de Toxicidade Comum.

\begin{tabular}{lllllll}
\hline & Grau 0 & Grau 1 & Grau 2 & Grau 3 & Grau 4 \\
\hline Leucopenia & Células brancas x 103 & $\geq 4$ & $3.0-3.9$ & $2.0-2.9$ & $1.0-1.9$ & $<1.0$ \\
& Granulócitos & $\geq 2$ & $1.5-1.9$ & $1.0-1.4$ & $0.5-0.9$ & $<0.5$ \\
& Linfócitos & $\geq 2$ & $1.5-1.9$ & $1.0-1.4$ & $0.5-0.9$ & $<0.5$ \\
\hline
\end{tabular}

final de radioterapia), e os estudos de Pieffert ${ }^{48}$ e Gerard ${ }^{43}$ mostraram taxas de resposta completa maiores, de $96 \%$ e $89 \%$, respectivamente, com o escalonamento da dose. No entanto, o estudo RTOG 92-08 ${ }^{53}$, que tratou 47 pacientes com dose final de radioterapia de 59,6 Gy, 5-FU e MMC, reportou taxas maiores de colostomia em dois anos $(30 \%$ versus $7 \%$ ), quando comparado com o RTOG 87-04 ${ }^{54}$ que utilizou o regime padrão de 45Gy, 5-FU e MMC. Embora a razão não esteja clara, os investigadores postulam que os resultados podem ser conseqüentes ao maior número de pacientes que exigiram suspensão temporária do tratamento maior que duas semanas no grupo de intensificação de dose (96\% versus $12 \%$ ). O estudo ECOG $4292^{55}$ submeteu 19 pacientes à intensificação de dose de radioterapia com 59,4 Gy, 5-FU e CDDP e observou resposta completa em somente $68 \%$ dos casos.

Enfim, ainda não é possível estabelecer nenhuma dose ideal de radioterapia. Em nossa série, observamos que doses menores que 45 Gy na pelve implicaram em menor controle local $(12,9 \%$ versus $87,1 \%, p=0,024)$.

O tratamento conservador do carcinoma de canal anal está relacionado à alta toxicidade aguda ${ }^{56,57}$ e à baixa toxicidade $\operatorname{tardia}^{58}$, como reportam as séries na literatura. Em nossa série, encontramos índices de toxicidade aguda e tardia similares à literatura. Apesar de Wong et al ${ }^{59}$ terem observado em sua série que a rápida proliferação tumoral não proporcionou impacto adverso no controle local, alguns estudos sugerem que o tempo de tratamento pode ser um fator significativo. Bartelink et $a l^{34}$ relatam que, no tratamento de carcinomas escamosos, cada dia de prolongamento da terapêutica exige acréscimo de dose ao plano original da ordem de 0,3 a $0,5 \mathrm{~Gy}$. Graf et $a l^{60}$ reportaram que em sua série de 111 pacientes tratados com esquema combinado, o tempo de tratamento global maior que 41 dias implicou em menor controle local ( $58 \%$ versus $79 \%, p=0,04)$. Resultados semelhantes foram observados na série reportada por Weber et $a l^{61}$, em que um período de pausa durante o tratamento maior que 37,5 dias resultou em menor controle local na análise uni e multivariada $(75 \%$ verus $92,3 \%$, $p=0,02$ ).

O estudo EORTC $22953^{62}$ testou a viabilidade em 44 pacientes de reduzir o período de pausa do tratamento entre a radiação pélvica e a complementação de dose no tumor primário para duas semanas, com 5-FU e MMC

Tabela 4 - Sobrevida global e livre de doença em 5 anos em função de variáveis clínicas e de tratamento.

\begin{tabular}{|c|c|c|c|c|c|}
\hline$\overline{\text { Categoria }}$ & $\begin{array}{c}\text { Freqüência } \\
\mathbf{n}^{0}(\%)\end{array}$ & \multicolumn{4}{|l|}{$\operatorname{Em} 5 \operatorname{anos}(\%)$} \\
\hline \multicolumn{6}{|c|}{ Estádio Clínico } \\
\hline $\mathrm{I}+\mathrm{II}$ & $30(63,8)$ & 69,5 & 0,0763 & 59,0 & 0,0543 \\
\hline IIIA + IIIB & $17(36,2)$ & 49,5 & & 33,4 & \\
\hline \multicolumn{6}{|c|}{ T (extensão) } \\
\hline $\mathrm{T} 1+\mathrm{T} 2$ & $33(70,2)$ & 63,2 & 0,3283 & 60,3 & 0,0111 \\
\hline $\mathrm{T} 3+\mathrm{T} 4$ & $14(29,8)$ & 59,8 & & 24,4 & \\
\hline \multicolumn{6}{|c|}{$\begin{array}{l}\text { Linfonodo Inguinal } \\
\text { clinicamente tumoral }\end{array}$} \\
\hline presente & $8(17)$ & 17,4 & 0,0085 & 22,5 & 0,0207 \\
\hline ausente & $39(83)$ & 70,7 & & 56,7 & \\
\hline \multicolumn{6}{|l|}{ RT pelve } \\
\hline$<45$ Gy & $11(23,4)$ & 42,4 & 0,0110 & 36,4 & 0,4406 \\
\hline$\geq 45 \mathrm{~Gy}$ & $36(76,6)$ & 67,7 & & 55,6 & \\
\hline \multicolumn{6}{|c|}{ Resposta completa } \\
\hline $\operatorname{sim}$ & $40(85,1)$ & 72,5 & $<0,001$ & 60,0 & $<0,001$ \\
\hline não & $7(14,9)$ & 0,0 & & 0,0 & \\
\hline \multicolumn{6}{|c|}{ Esfíncter preservado } \\
\hline $\operatorname{sim}$ & $38(80,9)$ & 63,2 & 0,8632 & 60,6 & $<0,001$ \\
\hline não & $9(19,1)$ & 55,6 & & 11,1 & \\
\hline \multicolumn{6}{|c|}{ Controle local } \\
\hline $\operatorname{sim}$ & $31(66)$ & 77,8 & $<0,001$ & 74,4 & $<0,001$ \\
\hline não & $16(34)$ & 31,3 & & 6,3 & \\
\hline
\end{tabular}


Tabela 5 - Características clínicas dos pacientes e do tratamento relacionadas com o controle local.

\begin{tabular}{|c|c|c|c|c|}
\hline Variável & $\begin{array}{c}\text { Freqüência } \\
\text { no }(\%)\end{array}$ & $\begin{array}{l}\text { Controle Local } \\
\text { Presente no }(\%)\end{array}$ & $\begin{array}{l}\text { Controle Local } \\
\text { Ausente no }(\%)\end{array}$ & Valor $\mathbf{p}$ \\
\hline \multicolumn{5}{|c|}{ Estádio Clínico } \\
\hline $\mathrm{I}+\mathrm{II}$ & $30(63,8)$ & $21(67,7)$ & $9(56,3)$ & 0,528 \\
\hline IIIA + IIIB & $17(36,2)$ & $10(32,3)$ & $7(43,7)$ & \\
\hline \multicolumn{5}{|c|}{ T (extensão) } \\
\hline $\mathrm{T} 1+\mathrm{T} 2$ & $33(70,2)$ & $23(74,2)$ & $10(62,5)$ & 0,506 \\
\hline $\mathrm{T} 3+\mathrm{T} 4$ & $14(29,8)$ & $8(25,8)$ & $6(37,5)$ & \\
\hline \multicolumn{5}{|c|}{$\begin{array}{l}\text { Linfonodo Inguinal } \\
\text { clinicamente tumoral }\end{array}$} \\
\hline presente & $8(17)$ & $4(12,9)$ & $4(25)$ & 0,416 \\
\hline ausente & $39(83)$ & $27(87,1)$ & $12(75)$ & \\
\hline \multicolumn{5}{|c|}{ LN inguinal bilateral tumoral } \\
\hline presente & $3(6,4)$ & $1 \quad(3,2)$ & $2(12,5)$ & 0,264 \\
\hline ausente & $44(93,6)$ & $30(96,8)$ & $14(87,5)$ & \\
\hline \multicolumn{5}{|l|}{ RT pelve } \\
\hline$<45 \mathrm{~Gy}$ & $11(23,4)$ & $4(12,9)$ & $7(43,8)$ & 0,024 \\
\hline$\geq 45 \mathrm{~Gy}$ & $36(76,6)$ & $27(87,1)$ & $9(56,2)$ & \\
\hline \multicolumn{5}{|c|}{ Resposta completa } \\
\hline $\operatorname{sim}$ & $40(85,1)$ & $31(100)$ & $9(56,3)$ & $<0,001$ \\
\hline não & $7(14,9)$ & $0 \quad(0,0)$ & $7(43,7)$ & \\
\hline
\end{tabular}

concomitantes. A comparação deste estudo com o EORTC 22861 revelou maior controle local ( $88 \%$ versus $68 \%$ ), maior intervalo livre de colostomia ( $81 \%$ versus $72 \%)$, maior intervalo livre de toxicidade tardia severa ( $84 \%$ versus $62 \%)$, e maior sobrevida global ( $81 \%$ versus $70 \%)$, o que tornou o estudo 22953 o novo padrão de tratamento para carcinoma do canal anal da EORTC. Apesar da necessidade de novos estudos aleatorizados fase III para estabelecer a real importância do tempo de tratamento, estes trabalhos sugerem que a diminuição no tempo de tratamento pode ser benéfica para o paciente. Portanto, a diminuição da toxicidade poderia reduzir o tempo de tratamento e proporcionar melhores índices terapêuticos. Na presente série, o tempo de tratamento não influenciou o controle local, a sobrevida global e a sobrevida livre de doença, utilizando-se valor de corte de 49 dias $(p=0,753, p=0,5533$ e $p=0,4351$, respectivamente).

As maiores séries ${ }^{29,33-35,40-43,48-52,63-72}$ de carcinoma de canal anal publicadas na literatura têm reportado incidência média de metástase inguinal sincrônica de $13 \%$ (variação de $6 \%$ a $23 \%$ ), com sobrevida global em cinco anos, considerando somente as séries mais recentes, de $48 \%$ (variação de $30 \%$ a $66 \%$ ). As terapias empregadas no tratamento da região inguinal variam de uma instituição para outra. Várias séries recomendam radioquimioterapia concomitantes ${ }^{40,69,71}$. Outras instituições preferem a linfadenectomia inguinal com rádio e quimioterapia pós-operatórias ${ }^{43,65,66,73}$. Gerard at a $l^{74}$ reportaram controle local de $86 \%$ após esta abordagem terapêutica. Na ausência de tratamento eletivo da região inguinal, as metástases inguinais metacrônicas incidem em $24 \%$ dos pacientes (variação de $16 \%$ a $36 \%$ ). Na série de Lyon, não trataram eletivamente a região inguinal em 243 pacientes, e a recidiva inguinal ocorreu em 19 pacientes $(7,8 \%)^{74}$. Estes pacientes foram submetidos à dissecção inguinal e radiotera- pia pós-operatória, com taxas de controle local e sobrevida global de $68 \%$ e $41,4 \%$, respectivamente. Várias instituições e os estudos aleatorizados de Flam $^{41}$ e do UKCCCR ${ }^{35}$ utilizaram a radioterapia inguinal eletiva. De La Rochefordiere ${ }^{69} \mathrm{e}$ outras instituições ${ }^{40,68-70}$ reportaram recidiva inguinal em menos de $5 \%$ dos pacientes irradiados eletivamente nessa região. Em nossa série, cinco pacientes $(11,6 \%)$ foram submetidos à radioterapia inguinal terapêutica e quatro tiveram a doença controlada nessa região. A radioterapia inguinal eletiva foi realizada em quatro pacientes e nenhum apresentou recidiva inguinal. Dos três pacientes EC IIIB, dois foram controlados com linfadenectomia. Um paciente EC II não recebeu RT inguinal e apresentou metástase inguinal metacrônica não tendo sido possível resgatá-lo com cirurgia ou RT. Um paciente ECIIIB recebeu tratamento primário com RT em região inguinal, porém, apresentou progressão de doença inguinal e o resgate cirúrgico não pode ser efetuado. A profilaxia da região inguinal necessita de ensaios com maior nível de evidência científica para estabelecer a melhor abordagem nos pacientes com carcinoma de canal anal, mas os resultados de literatura e os encontrados em nossa série sugerem que pacientes com tumores $\mathrm{T} 2$ ou maiores se beneficiam da radioterapia eletiva da região inguinal. Em nossa série, a sobrevida global e sobrevida livre de doença em cinco anos para os pacientes com linfonodo inguinal tumoral foi de $17,4 \%$ e $22,5 \%$, respectivamente ( $p=0,0085$ e $p=0,0207)$. Nenhum paciente com linfonodo inguinal tumoral bilateral apresentavase vivo em dois anos.

Estudos sobre o estadiamento com dissecção do linfonodo sentinela têm-se mostrado favoráveis. Mistrangelo et $a l^{75}$ realizaram a procura pelo linfonodo sentinela em uma paciente, e identificaram linfonodos inguinais bilateralmente acometidos. Gerard ${ }^{74}$ abordou 13 pacientes com esta técnica e 
identificou envolvimento linfático microscópico em dois pacientes. Peley et $a l^{76}$ submeteram oito pacientes à dissecção do linfonodo sentinela e dois tinham metástases linfáticas. Estudos com maior nível de evidência científica são necessários, mas o adequado estadiamento da região inguinal pode diminuir a morbidade relacionada ao tratamento, visto que, em média, $63 \%$ dos pacientes não serão acometidos por metástases inguinais ${ }^{74}$ e mortes relacionadas à toxicidade do tratamento podem acontecer em $2 \%$ a $2,7 \%$ dos pacientes ${ }^{34,35,41}$.

Seguindo a filosofia de diminuição de toxicidade do tratamento, seja para melhorar o controle local ou diminuir as mortes relacionadas à terapia ${ }^{34,35,41}$, as novas técnicas conformadas e, especialmente a radioterapia de intensidade modulada, podem trazer grandes benefícios para o tratamento do carcinoma de canal anal, especialmente para os pacientes HIV positivos, os quais, em relação aos pacientes HIV negativos, têm menor tolerância ao tratamento combinado $(80 \%$ versus $30 \%$ de toxicidade, $p<0,005)$ e menor tempo para morte relacionada ao câncer $(1,4 \text { anos versus 5,3 anos, } p<0,05)^{77}$. No estudo fase II reportado por Vuong $\mathrm{T}$ et $a l^{7}, 30$ pacientes foram tratados com 54 Gy em 30 frações, com dois ciclos de 5-FU e MMC com técnica conformada. Todos os pacientes terminaram o tratamento sem pausas, com baixas taxas de toxicidades cutâneas, intestinais e em medula óssea (20\%, 3,3\% e 13,3\%, respectivamente). Com seguimento mediano de 33 meses, a taxa de sobrevida livre de recidiva em quatro anos foi de $91 \%$.

Em nossa série, o índice de metástases à distância foi relativamente alto (14,9\%). A sobrevida global $(61,5 \%)$ e a sobrevida livre de doença $(50,1 \%)$ em cinco anos foram semelhantes a algumas séries da literatura, porém, não ideais. Provavelmente, estas taxas ocorreram em função de nossa amostra ser composta por 36,2\% de pacientes ECIII. Ensaios clínicos bem controlados podem ser opções adequadas para esse grupo de pacientes, especialmente os pacientes ECIIIB, com a utilização de novos agentes quimioterápicos para controlar a doença metastática.

O carcinoma de canal anal é intimamente relacionado com a infecção pelo papiloma vírus humano. Frish et $a l^{79}$ conduziram um estudo populacional caso controle na Dinamarca e na Suécia, em que 417 pacientes com câncer do canal anal, 534 controles com adenocarcinoma de reto, e 554 controles da população geral foram analisados para diversos fatores comportamentais, e, na análise uni e multivariada, a maior quantidade de relações sexuais foi relacionada com maior risco de câncer de canal anal, e a infecção pelo papiloma vírus humano foi presumida pelos autores como causa etiológica ${ }^{7,79}$. Há, também, fortes suspeitas de que o papiloma vírus humano associado ao câncer de colo uterino seja similar ao subtipo relacionado com o desenvolvimento de neoplasias intraepiteliais anais, as quais são precursoras imediatas do câncer anal ${ }^{7,80-82}$. Com as pesquisas voltadas para o desenvolvimento de vacinas contra o papiloma vírus humano do câncer de colo uterino, provável e felizmente, o câncer de canal anal tornar-se-á ainda mais raro.

Como na série anterior do Centro de Tratamento e Pesquisa Hospital do Câncer reportada por Lima et $a l^{24}$, concluímos que o tratamento padrão atual do carcinoma do canal anal, baseado na literatura e confirmado por esta série, é o regime combinado de rádio e quimioterapia, com a cirurgia reservada para os casos de recidiva local ou respostas não completas ao tratamento inicial, oferecendo ao paciente uma chance real de cura e preservação da função esfincteriana. Novas drogas e novas técnicas de radioterapia são promissoras no sentido de diminuir a toxicidade do tratamento, diminuir a chance de interrupção do mesmo e propiciar níveis de cura e qualidade de vida satisfatória para os pacientes que são acometidos pelo carcinoma do canal anal.

\begin{abstract}
Background: To report the results of conservative treatment in patients with anal canal carcinoma with radiotherapy and chemotherapy at Centro de Tratamento e Pesquisa Hospital do Câncer A. C. Camargo. Methods: From March 1993 to December 2001, 47 patients with histological diagnosis of anal canal carcinoma were treated by conservative treatment. The median dose of radiotherapy at whole pelvis and at primary tumor was $45 G y$ and $55 G y$, respectively. The chemotherapy regimen was provided with 5-Fluorouracil and Mitomycin C, with median doses of $1000 \mathrm{mg} / \mathrm{m}^{2}$ and $10 \mathrm{mg} / \mathrm{m}^{2}$, respectively. Thirty-eight (80.8\%) patients didn't receive radiotherapy at inguinal region. The median follow-up period was 40 months (8 days-116 months). Results: Complete responses were observed in 40 patients (85.1\%). The local control was obtained in 38 patients (80.9\%). Distant metastases were observed in $7(14.9 \%)$ patients. The overall survival and colostomy and disease-free survival in 5 years were $61,5 \%$ and 50,1\%, respectively. The overall survival and disease free survival in 5 years for patients that obtained local control were $77,8 \%(\mathrm{p}<0.001)$ and $74,4 \%(\mathrm{p}<0.001)$. The overall survival and disease free survival in 5 years for patients with clinical inguinal tumoral lymph nodes were $70.7 \%$ and $56.7 \%$ respectively $(\mathrm{p}=0.0085$ and $\mathrm{p}=0.0207)$. Twelve $(28 \%)$ patients required temporary interruption of treatment. Five patients had mild chronic complications. Conclusions: The treatment scheme of these patients was effective for anal sphincter preservation and local control of the disease. The presence of clinical tumoral inguinal lymph nodes and the local control were the main prognostic factors for overall and disease free survival. The relative elevated recurrence at inguinal region suggests the need of elective radiotherapy at this region. The acute toxicity was elevated.
\end{abstract}

Key words: Carcinoma; Anus; Anus neoplasms; Radiotherapy; Chemotherapy.

\section{REFERÊNCIAS}

1. Stearns MW Jr, Urmacher C, Sternberg SS, et al. - Cancer of the anal canal. Curr Probl Cancer,1980,4(12):1-44.

2. Landis SH, Murray T, Bolden S, et al.- Cancer statistics, 1999. CA Cancer J Clin, 1999,49(1):8-31
3. Myerson RJ, Karnell LH, Menck HR - The National Cancer Data Base report on carcinoma of the anus. Cancer, 1997,80(4):805-815.

4. Klas JV, Rothenberger DA, Wong WD, et al. - Malignant tumors of the canal anal: the spectrum of disease, treatment and outcomes. Cancer, 1999,85(8):1686-1693. 
5. Nadalin W, Aguilar PB, Habr-Gama A, et al. - Tumores colorretal e canal anal. In Salvajoli JV, Souhami L, Faria SL, (eds) - Radioterapia em oncologia. São Paulo. Medsi, 1999, pp. 583-600.

6. Minsky BD - Anal canal cancer. In Leibel AS, Phillips T (eds) Textbook of radiation oncology. Philadelphia. WB Saunders, 1998, pp. 703-709.

7. Minsky BD, Hoffman JP, Kelsen DP - Cancer of the anal region. In DeVita Jr VT, Hellman S, Rosenberg SA (eds) - Cancer: principles $\&$ practice of oncology. $6^{\text {th }}$ Edition. Philadelphia. Lippincott-Williams \& Wilkins, 2001, pp.1319-1342.

8. Morson BC - The pathology and results of treatment of squamous cell carcinoma of the anal canal and anal margin. Proc R Soc Med, 1960,53:414.

9. Greenall MJ, Quan SH, Stearns MW, et al. - Epidermoid cancer of the anal margin. Pathologic features, treatment, and clinical results. Am J Surg, 1985,149(1):95-101

10. Hardy KJ, Hughes ES, Cuthbertson AM - Squamous cell carcinoma fo the anal canal and anal margin. Aust N Z J Surg, 1969,38(4):301-305.

11. Al-Jurf AS, Turnbull RP, Fazio VW - Local treatment of squamous cell carcinomaof the anus. Surg Gynecol Obstet, 1979,148(4):576-578

12. Beahrs O, Wilson SM - Carcinoma of the anus. Ann Surg, 1976,184(4):422-428

13. Kuehn PG, Beckett R, Eisenberg H - Hematogenous metastasis from epidermoid carcinoma of the anal canal. Am J Surg, 1965,109:455.

14. Grodsky L - Unsuspected anal cancer discovered after minor anorectal surgery. Dis Colon Rectum,1967,10(6):471-478.

15. American Joint Committee on Cancer. Anal cancer. In Fleming ID, Cooper JS, Henson DE, et al. (eds) - AJCC cancer staging manual. Philadelphia. Lippincott-Raven, 1998, pp. 91.

16. Anal canal. In Sobin LH, Wittekind CH (eds) - TNM classification of malignat tumours. New York. Wiley-Liss, 1997, pp. 70.

17. American Joint Committee on Cancer. Anal cancer. In Greene FL, Page DL, Fleming ID, et al. (eds) - AJCC cancer staging manual. Springer-Verlag. 2001, pp.139.

18. Anal canal. In Sobin LH, Wittekind CH (eds) - TNM classification of malignat tumours. New York. Wiley-Liss, 2002, pp.77.

19. Nigro ND, Vaitkevicius VK, Considine B - Combined therapy for cancer of the anal canal: a preliminary report. Dis Colon Rectum, 1974,17(3):354-356.

20. Habr-Gama A, Sousa Jr. AHS, Nadalin W, et al - Epidermoid carcinoma of the anal canal. Results of treatment by combined chemotherapy and radiation therapy. Dis Colon Rectum, 1989,32(9):773-777.

21. Martenson Jr. JA, Schild SE, Haddock MG - Cancers of the gastrointestinal tract. In Khan FM, Potish RA (eds) - Treatment planning in radiation oncology. Baltimore. Williams \& Wilkins, 1998, pp. 319-342.

22. Martenson Jr. JA, Haddock MG, Gunderson LL - Cancers of the colon rectum, and anus. In Levitt SH, Potish RA, Khan FM, et al. (eds) - Technological basis of radiation therapy. Philadelphia. Lippincott Williams \& Wilkins, 1999, pp. 335-347.

23. Kalend AM, Park TL, Wu A, et al. - Clinical use of a wing field with transmission block for the treatment of the pelvis including the inguinal node. Int J Radiat Oncol Biol Phys, 1990,19(1):153-158.

24. Lima Jr. CGB, Ferrigno R, Salvajoli JV, et al. -Tratamento do carcinoma do canal anal com radioterapia e quimioterapia concomitantes. Resultados preliminares do Hospital do Câncer A. C. Camargo. Rev Col Bras Cir, 2000,28(1):30-38.

25. Ferrigno R, Edna Andrade da Cruz - Tumores do canal anal e reto. In Pellizzon ACA, Salvajoli JV, Maia MAC, et al. (eds) - Rotinas e condutas em radioterapia. São Paulo. Lemar,2002, pp. 95100.
26. Cox JD, Stetz J, Pajak TF - Toxicity criteria of the Radiation Therapy Oncology Group (RTOG) and the European Organization for Research and Treatment of Cancer (EORTC). Int J Radiat Oncol Biol Phys, 1995,31(5):1341-1346.

27. Oken MM, Creech RH, Tormey DC, et al. - Toxicity and response criteria of the Eastern Cooperative Oncology Group. Am J Clin Oncol,1982,5(6):649-655.

28. Kaplan EL, Meier P- Non parametric estimation from incomplete observation. J Am Stat Assoc, 1958,53:457-481.

29. Boman BM, Moertel CG, O’Connell MJ, et al. - Carcinoma of the anal canal. A clinical and pathologic study of 188 cases. Cancer, 1984,54(1):114-125.

30. Beck DE, Wexner SD. Anal neoplasms. In Beck DE, Wexner SD (eds) - Fundamentals of anorectal surgery. $2^{\text {nd }}$ Edition. London, WB Saunders, 1998, pp. 261-277.

31. Buroker TR, Nigro N, Bradley G, et al. - Combined therapy for cancer of the anal canal: a follow-up report. Dis Colon Rectum, 1977,20(8):677-678.

32. Nigro ND - An evaluation of combined therapy for squamous cell cancer of the anal canal. Dis Colon Rectum, 1984,27(12):763-766.

33. Touboul E, Schlienger M, Buffat L, et al. - Epidermoid carcinoma of the anal canal. Results of curative-intent radiation therapy in a series of 270 patients. Cancer, 1994,73(6):1569-1579.

34. Bartelink H, Roelofsen F, Eschwege F, et al. - Concomitant radiotherapy and chemotrerapy is superior to radiotherapy alone in the treatment of locally advanced anal cancer: results of a phase III randomized trial of the European Organization for Research and Treatment of Cancer Radiotherapy and Gastrointestinal Cooperative groups. J Clin Oncol, 1997,15(5):2040-2049.

35 .Epidermoid anal cancer: results from the UKCCCR randomised trial of radiotherapy alone versus radiotherapy, 5-fluorouracil, and mitomycin. UKCCCR anal Cancer Trial Working Party. Lancet,1996,348(9034):1049-1054.

36. Colvin OM - Antitumor Alkylating Agents. In DeVita VT, Hellman S, Rosenberg SA (eds) - Cancer: principles \& practice of oncology. $6^{\text {th }}$ Edition. Philadelphia. Lippincott-Williams \& Wilkins, 2001, pp. 363-376

37. Liss AP, Luedke S, Einhorn L, et al. - Vindesine and mitomycin $\mathrm{C}$ in metastatic breast cancer. A Southeastern Cancer Study Group Trial. Oncology, 1989,46(6):357-359.

38. Hong RL, Sheen TS, Ko JY, et al. - Induction with mitomycin C, doxorubicin, cisplatin and maintenance with weekly 5fluorouracil, leucovorin for treatment of metastatic nasopharyngeal carcinoma: a phase II study. Br J Cancer, 1999,80(12):1962-1967

39. Arbuck SG, Silk Y, Douglass HO Jr, et al. - A phase II trial of5fluorouracil, doxorubicin, mitomycin $\mathrm{C}$, and leucovorin in advanced gastric carcinoma. Cancer, 1990,65(11):2442-2445.

40. Cummings BJ, Keane TJ, O’Sullivan B, et al. - Epidermoid anal cancer: treatment by radiation alone or by radiation and 5fluorouracil with and without mitomycin C. Int J Radiat Oncol Biol Phys, 1991,21(5):1115-1125.

41. Flam M, John M, Pajak TF, et al. - Role of mitomycin in combination with fluorouracil and radiotherapy, and of salvage chemoradiation in the definitive nonsurgical treatment of epidermoid carcinoma of the anal canal: results of a phase III randomized intergroup study. J Clin Oncol, 1996,14(9):2527-2539.

42. Sandhu AP, Symonds RP, Robertson AG, et al. - Intersticial iridium-192 implantation combined with external radiotherapy in anal cancer: ten years experience. Int J Radiat Oncol Biol Phys, 1998,40(3):575-581.

43. Gerard JP, Ayzac L, Hun D, et al. - Treatment of anal canal carcinoma with high dose radiation therapy and concomitant fluorouracil-cisplatinum. Long-term results in 95 patients. Radiother Oncol, 1998,46(3):249-256. 
44. Lohnert M, Doniec JM, Kovacs G, et al. - New method of radiotherapy for anal cancer with three-dimensional tumor reconstruction based on endoanal ultrasound and ultrasound-guided afterloading therapy. Dis Colon Rectum, 1998,41(2):169-176.

45. Papillon J, Montbarbon JF - Epidermoid carcinoma of the anal canal. A series of 276 cases. Dis Colon Rectum, 1987,30(5):324-333.

46. Roed H, Engelholm SA, Svendsen LB, et al. - Pulsed dose rate (PDR) brachytherapy of anal carcinoma. RadiotherOncol,1996,41(2):131-134.

47. Allal AS, Mermillod B, Roth AD, et al. - Impact of clinical and therapeutic factors on major late complications after radiotherapy with or without concomitant chemotherapy for anal carcinoma. Int J Radiat Oncol Biol Phys,1997,39(5):1099-1105.

48. Peiffert D, Bey P, Pernot M, et al. - Conservative treatment by irradiation of epidermoid cancers of the anal canal: prognostic factors of tumoral control and complications. Int J Radiat Oncol Biol Phys, 1997,37(2):313-324.

49. Wagner JP, Mahe MA, Romestaing P, et al. - Radiation therapy in the conservative treatment of carcinoma of the anal canal. Int J Radiat Oncol Biol Phys, 1994,29(1):17-23.

50. Doci R, Zucali R, Monica G, et al. - Primary chemoradiation therapy with fluorouracil and cisplatin for cancer of the anus: results in 35 consecutive patients. J Clin Oncol, 1996,14(12):3121-3125.

51. Constantinou EC, Daly W, Fung CY, et al. - Time-dose considerations in the treatment of anal cancer. Int J Radiat Oncol Biol Phys, 1997,39(3):651-657.

52. Hughes LL, Rich TA, Delclos L, et al. - Radiotherapy for anal cancer: experience from 1979-1987. Int J Radiat Oncol Biol Phys, 1989,17(6):1153-1160.

53. John M, Pajak T, Flam M, et al. - Dose Escalation In Chemoradiation For Anal Cancer: Preliminary Results Of RTOG 92-08. Cancer J Sci Am, 1996,2(4):205.

54. Flam MS, John MJ, Peters T, et al. - Radiation and 5-fluorouracil $(5 \mathrm{FU})$ vs. radiation, $5 \mathrm{FU}$, mitomycin-C (MMC) in the treatment of anal canal carcinoma: preliminary results of a phase III randomized RTOG/ECOG intergroup trial. Am J Clin Oncol, 1993,12:192.

55. Marterson JA, Lipsitz SR, Wagner H, et al. - Initial results of a phase II trial of of high dose radiation therapy, 5-fluorouracil, and cisplatin for patients with anal cancer (E4292): an Eastern Cooperative Oncology Group study. Int J Radiat Oncol Biol Phys, 1996,35(4):745-749.

56. Tanum G, Tveit KM, Karlsen KO - Chemoradiotherapy of anal carcinoma: tumour response and acute toxicity. Oncology,1993, 50(1):14-17.

57. Mitchell SE, Mendenhal WM, Zlotecki RA, et al. - Squamous cell carcinoma of the anal canal. Int J Radiat Oncol Biol Phys, 2001,49(4):1007-1013.

58. Allal AS, Sprangers MA, Laurencet F, et al. - Assessment of long-term quality of life in patients with anal carcinomas treated by radiotherapy with or without chemotrerapy. Br J Cancer, 1999,80(10):1588-1594.

59. Wong CS, Tsang RW, Cummings BJ, et al. - Proliferation parameters in epidermoid carcinomas of the anal canal. Radiother Oncol, 2000,56(3):349-353.

60. Graf R, Wust P, Hildebrandt B, et al. - Impact of overall treatment time on local control of anal cancer treated with radiochemotherapy. Oncology, 2003,65(1):14-22.

61. Weber DC, Kurtz JM, Allal AS - The impact of gap duration on local control in anal canal carcinoma treated by split-course radiotherapy and concomitant chemotherapy. Int J Radiat Oncol Biol Phys, 2001,50(3):675-680.

62. Bosset JF, Roelofsen F, Morgan DA, et al. - Shortened irradiation scheme, continuous infusion of 5-fluorouracil and fractionation of mitomycin $\mathrm{C}$ in locally advanced anal carcinomas. Results of a phase II study of the European Organization for Research and Treatment of Cancer. Radiotherapy and Gastrointestinal Cooperative Groups. Eur J Cancer, 2003,39(1):45-51.

63. Stearns MV, Quan SH - Epidermoid carcinoma of the anorectum. Surg Gynecol Obstet,1970,131(5):953-957.
64. James RD, Pointon RS, Martin S - Local radiotherapy in the management of squamous carcinoma of the anus. Br J Surg, 1985,72(4):282-285.

65. Papillon J, Mayer M, Montbarbon JF, et al. - A new approach to the management of epidermoid carcinoma of the anal canal. Cancer,1983,51(10):1830-1837.

66. Allal AS, Laurencet FM, Reymond MA, et al. - Effectiveness of surgical salvage therapy for patients with locally uncontrolled anal carcinoma after sphincter-conserving treatment. Cancer,1999,86(3):405-409.

67. Myerson RJ, Shapiro SJ, Lacey D, et al. - Carcinoma of the anal canal. Am J Clin Oncol, 1995,18(1):32-39.

68. Tanum G, Tveit K, Karlsen KO, et al. - Chemotherapy and radiation therapy for anal carcinoma. Survival and late morbidity. Cancer,1991,67(10):2462-2466.

69. De La Rochefordiere A, Pontvert D, Asselain B, et al. Radiothérapie des cancers du canal anal. Expérience de l'Institut Curie dans le traitement des aires ganglionnaires. Bull Cancer Radiother, 1993,80:391-398.

70. Grabenbaur GG, Matzel KE, Schneider IH, et al. - Sphincter preservation with chemoradiation in anal canal carcinoma: abdominoperineal resection in selected cases? Dis Colon Rectum,1998,41(4):441-450.

71. Newman G, Calverley DC, Alker BD, et al. - The management of carcinoma of the anal canal by external beam radiotherapy, experience in Vancouver 1971-1988. Radiother Oncol, 1992,25(3):196-202.

72. Lusinchi $\mathrm{A}$, Wang $\mathrm{C}$, Wibault $\mathrm{P}$, et al. - Radiothérapie à visée conservatrice des cancers du canal anal: expérience de l'IGR. Bull Cancer Radiother, 1993,80:400.

73. Schlienger M, Krzisch C, Pene F, et al. - Epidermoid carcinoma of the anal canal: treatment results and prognostic variables in a series of 242 cases. Int J Radiat Oncol Biol Phys, 1989,17(6):1141-1151.

74. Gerard JP, Chapet O, Samiei F, et al. - Management of inguinal lymph node metastases in patients with carcinoma of the anal canal: experience in a series of 270 patients treated in Lyon and review of the literature. Cancer,2001,92(1):77-84.

75. Mistrangelo M, Mobiglia A, Mussa B, et al. - The sentinel node in anal carcinoma. Tumori, 2002,88(3):S51-52.

76. Peley G, Farkas E, Sinkovics I, et al. - Inguinal sentinel lymph node biopsy for staging anal cancer. Scand J Surg, 2002,91(4):336-338.

77. Kim JH, Sarani B, Orkin BA, et al. - HIV-positive patients with anal carcinoma have poorer treatment tolerance and outcome than HIV-negative patients. Dis Colon Rectum, 2001,44(10):1496-502.

78. Vuong T, Devic S, Belliveau P, et al. - Contribution of conformal therapy in the treatment of anal canal carcinoma with combined chemotherapy and radiotherapy: results of a phase II study. Int J Radiat Oncol Biol Phys, 2003,56(3):823-831.

79. Frisch M, Fenger C, van den Brule AJ, et al. - Variants of squamous cell carcinoma of the anal canal and perianal skin and their relation to human papillomaviruses. Cancer Res, 1999,59(3):753-757.

80. Friedman HB, Saah AJ, Sherman ME, et al. - Human papillomavirus, anal squamous intraepithelial lesions, and human immunodeficiency virus in a cohort of gay men. J Infect Dis, 1998,178(1):45-52

81. Palefsky JM, Holly EA, Ralston ML, et al. - High incidence of anal high-grade squamous intra-epithelial lesions among HIVpositive and HIV-negative homosexual and bisexual men. AIDS, 1998,12(5):495-503.

82. Scholefield JH, Hickson WG, Smith JH, et al. - Anal intraepithelial neoplasia: part of a multifocal disease process. Lancet, 1992,340(8830):1271-1273.

Endereço para correspondência:

Ricardo Akiyoshi Nakamura

Rua Apeninos, 236, apto 82 - 01533-000

Paraíso- São Paulo

E-mail: ricardonakamura1@yahoo.com.br 